\title{
Phenylalanine and tyrosine measurements across gestation by tandem mass spectrometer on dried blood spot cards from normal pregnant women
}

\author{
Kim L. McBride, MD, MS ${ }^{1,2,3}$, Jill Pluciniczak, $\mathrm{MD}^{1,5}$, Timothy Rhyand, $\mathrm{BSC}^{4}$ and \\ Dennis Bartholomew, MD ${ }^{1,3}$
}

Purpose: Maternal phenylketonuria (MPKU) requires strict control of phenylalanine (Phe) and supplemental tyrosine (Tyr). Monitoring during pregnancy using dried blood spot (DBS) cards by tandem mass spectrometry (MS/MS) is now standard practice, however there are no Phe and Tyr reference ranges for DBS MS/MS method in healthy pregnant women.

Methods: DBS cards (63-1364 days in storage) from healthy women with singleton pregnancies were analyzed by MS/MS. Three hundred ninety DBS cards from 170 pregnancies (5/1-39/6 weeks' gestation), were tested.

Results: Both Phe and Tyr levels declined from the first trimester (Phe: $36.2+/-10.6$; Tyr $25.7+/-9.7 \mu \mathrm{mol} / \mathrm{L}$ ) to the second trimester (Phe 33.4+/-9.3; Tyr $21.7+/-6.7 \mu \mathrm{mol} / \mathrm{L}$ ) and remained stable in the third trimester (Phe $32.3+/-8.7$; Tyr $21.0+/-$ $6.6 \mu \mathrm{mol} / \mathrm{L}$ ). Phe and Tyr levels declined over time since collection
(Phe: $0.004 \mu \mathrm{mol} / \mathrm{L}$ per day; Tyr $0.002 \mu \mathrm{mol} / \mathrm{L}$ ). Nomograms by gestational age were created using raw data and data adjusted for time from sample collection. Reference ranges by trimester are provided.

Conclusions: Both Phe and Tyr decline quickly during the first trimester and remain relatively constant over the second and third trimesters. These nomograms will provide a valuable resource for care of MPKU.

Genetics in Medicine (2019) 21:1821-1826; https://doi.org/10.1038/s41436018-0407-8

Keywords: phenylketonuria; reference values; amino acids; inborn error of metabolism; pregnancy/metabolism

\section{INTRODUCTION}

Phenylketonuria (PKU, OMIM 26160) is an autosomal recessive inborn error of metabolism caused by pathogenic variants in the gene encoding the enzyme phenylalanine hydroxylase (PAH, OMIM 612349). This enzyme metabolizes the amino acid phenylalanine (Phe) to tyrosine (Tyr). Untreated individuals with PKU develop neurological disease, including developmental delay, seizures, intellectual disability, and psychiatric disorders, due to extremely elevated levels of Phe in their blood and tissues. ${ }^{1}$ Therapy consists of lifelong Phe restriction through dietary modification. In addition, high Phe levels during pregnancy are extremely teratogenic to the developing fetus. Untreated maternal PKU (MPKU) can cause neurological deficits and birth defects in the fetus (termed MPKU syndrome) due to antenatal exposure to elevated Phe levels. ${ }^{2}$ Therefore, dietary Phe restriction is particularly important during pregnancy because maintaining maternal Phe levels between 2 and $6 \mathrm{mg} / \mathrm{dL}(120-360 \mu \mathrm{mol} / \mathrm{L})$ prevents the fetal complications of untreated MPKU. Tyrosine is normally considered a nonessential amino acid because it can be generated from Phe. However, in PKU, Tyr becomes an essential amino acid because the inherent defect prevents $\mathrm{Tyr}$ from being generated. As such, dietary supplementation of Tyr becomes necessary for individuals with PKU. Maintaining normal Tyr levels are important, as Tyr serves as a precursor for catecholamines, thyroid hormones, and melanin. While monitoring of Phe levels for MPKU is vital, monitoring of Tyr levels is also important because their levels may have health implications as well. Theoretically both low or high tyrosine levels may be potentially harmful, with low Tyr causing poor fetal growth or pregnancy loss and neurologic deficits., ${ }^{3,4}$

Serum analyte measurements during pregnancy frequently vary compared with nonpregnant women. Several previous studies have looked at normal serum or plasma amino acid levels during pregnancy using amino acid analyzers, noting lower levels in the second trimester and third trimester. ${ }^{5-9}$

${ }^{1}$ Division of Genetic and Genomic Medicine, Nationwide Children's Hospital, Columbus, OH 43205, USA; ${ }^{2}$ Center for Cardiovascular Research, Nationwide Children's Hospital,
Columbus, OH, USA; ${ }^{3}$ Department of Pediatrics, College of Medicine, The Ohio State University, Columbus, OH, USA; ${ }^{4}$ Department of Laboratory Medicine, Nationwide Children's Hospital, Columbus, OH, USA; ${ }^{5}$ Present address: Mount Carmel Health, Columbus, OH, USA. Correspondence: Kim L. McBride (kim.mcbride@nationwidechildrens.org) 
However, these studies had limited sample size and did not assess trends over multiple time points during pregnancy. Two recent metabolomics studies have measured a variety of amino acids (including Phe and Tyr) on serum samples from pregnant women, using liquid chromatography coupled with mass spectrometry and proton nuclear magnetic resonance (1H-NMR) techniques. ${ }^{10,11}$

The recent trend in monitoring Phe and Tyr levels has been a simple dried blood spot (DBS) on a filter paper card, which can be easily collected and quickly and cheaply analyzed by tandem mass spectrometry (MS/MS). Previous studies of blood spot samples from normal nonpregnant individuals has shown MS/MS Phe measurements are approximately 15-25\% lower than the levels obtained with serum sample run on an amino acid analyzer (AAA). ${ }^{12,13}$ Variability in the values occur due to changes in hematocrit, thus blood spot card samples from pregnant women, when the hematocrit is lower than nonpregnant women, would be greater. Existing values from amino acid analyzers on serum samples thus cannot be used directly to interpret Phe and Tyr levels derived from blood spot samples run on MS/MS instruments, because they may suggest MS/MS levels are too low and lead to overtreatment. No studies have been published using a MS/MS instrument to analyze Phe and Tyr on blood spot samples from pregnant women. Thus the data available are sparse and not suited to modern practice using blood spot cards.

The purpose of this study was to investigate Phe and Tyr levels among normal pregnant women using MS/MS methods on blood spot card samples, to establish nomograms that can be used to guide therapy for MPKU.

\section{Ethics}

\section{MATERIALS AND METHODS}

This study was conducted using previously collected and deidentified samples. This study was reviewed by the Institutional Review Board at Nationwide Children's Hospital who deemed this nonhuman subject research, and no approval was required.

\section{Sample description}

DBS filter card samples were obtained from the Ohio Perinatal Research Network (OPRN; https://www.nationwidechildrens. org/research/areas-of-research/center-for-perinatal-research/ ohio-perinatal-research-network). This registry has collected samples from pregnant women, with every attempt for collection during the first, second, and third trimesters, as well as a postpartum sample. There were no exclusions of pregnancies for OPRN collection, and every attempt was made to record an accurate gestational age and any maternal and/or fetal complications of the current pregnancy. Because our goal was to attempt to create normal reference ranges of phenylalanine and tyrosine levels throughout pregnancy, our inclusion criteria included all DBS filter cards of singleton pregnancies that were able to collected in at least two trimesters with accurate gestational dating. Our exclusion criteria included any pregnancies that were complicated by the following conditions: pregestational and/or gestational diabetes mellitus, chronic and/or gestational hypertension, pre-eclampsia and/or eclampsia, intrauterine fetal growth restriction, and preterm labor/delivery.

DBS were prepared per the methodology of the OPRN. Blood was spotted onto filter paper and allowed to completely soak through the filter paper, which was then left to dry for 30 minutes. Cards were placed in a blood spot card storage box in a $-80^{\circ} \mathrm{C}$ freezer. Prior to use for this study, DBS cards were visually inspected to ensure they were correctly spotted, and a 3-mm punch was used to obtain a sample for analysis (performed in duplicate).

\section{Phenylalanine and tyrosine measurement}

Phe and Tyr measurements were performed on a Waters 2795 separation module liquid chromatography/Waters Quattro Micro quadrupole tandem mass spectrometer (Waters Corporation, Milford, MA). DBS samples were extracted using methanol that contained L-Phenylalanine $\left({ }^{13} \mathrm{C}_{6}\right)$ and L-Tyrosine $\left({ }^{13} \mathrm{C}_{6}\right)$ as internal standards, derivatized to their butyl ester forms with acidified n-butanol/n-butyl acetate, and analyzed using flow-injection analysis MS/MS. The values were determined using MassLynx software (Waters Corporation) to process the data and calculate the ratio of the intensity of the mass spectral peak of the sample to the intensity of the mass spectral peak of the internal standard with stable isotope. All runs were performed in duplicate with three quality control (QC) samples of low/ normal, high, and very high Phe and Tyr.

\section{Statistical analysis}

Summary statistics and hypothesis testing were performed using Stata 13.0 (StataCorp, College Station, Texas). Continuous variables were compared by $t$ test, regression, or analysis of variance (ANOVA), with a $p$ value $<0.05$ used for significance level. We also performed analysis using trimesters because this is a clinically intuitive grouping.

Equations from regression analysis were created using time from collection to analysis for each analyte, which were then used to generate a time collection adjusted set of values for Phe and Tyr. LMSChartMaker Light (version 2.54, Medical Research Council, UK) was used to create nomograms. The default settings for L (power transformation), M (median), and $S$ (coefficient of variation) were used, and then adjusted to obtain smooth curves over the gestational age, paying attention to changes in deviation as the models were fitted. The fitted values were then used to construct nomograms.

Reference ranges by trimester were created for both Phe and Tyr. Lower and upper limits of normal were defined as encompassing from $2.5 \%$ to $97.5 \%$ of values.

Sample size estimates for creating the reference centile curves were obtained from calculations in the paper by Jennen-Steinmetz using Table $\mathbf{1}$ (ref. ${ }^{14}$ ). Assumptions used were normal distribution of values (or transformation of data to normal) with homoscedasticity, symmetric accuracy on a logit scale, and 95th quantile with modest accuracy tolerance 


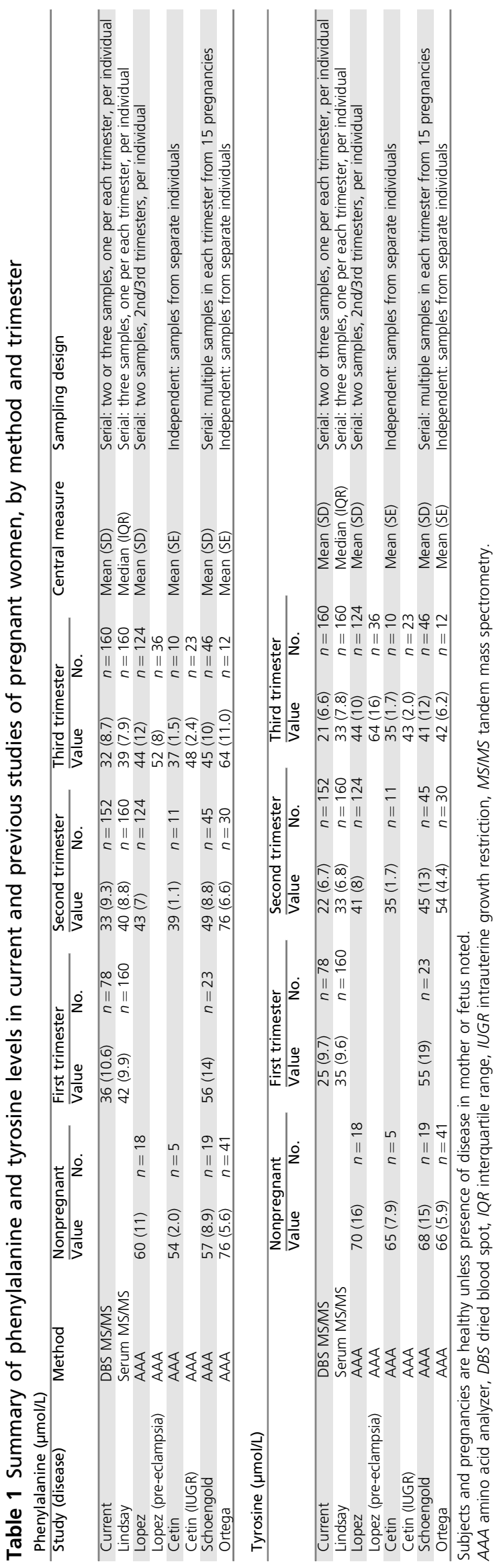

(quantile captures $0.914-0.972$ with a power of 0.90 ). These assumptions indicate a need for at least 143 measurements, with up to 309-364 measurements if we anticipate some heteroscedasticity of the distribution.

\section{RESULTS}

A total of 170 pregnancies, with 390 DBS filter card samples, were tested. Fifty-two of these pregnancies provided samples from all three trimesters, and 118 provided samples from two trimesters. The gestational age range of the samples was 5/1-39/6 weeks' gestation, with 78 samples within the first trimester (0-11/6 weeks' gestation), 152 samples within the second trimester (12/0-23/6 weeks' gestation), and 160 within the third trimester (24/0 weeks' gestation-delivery). Samples were analyzed from 63 to 1364 days after collection (mean $901+/-350$; median 1000). Measurements with comparison to literature are noted in Table $\mathbf{1 .}$

A repeated measures ANOVA was performed initially and no statistically significant repeated measures effect was found for either Phe or Tyr. No difference was noted for the mean and standard deviation for either analyte for any of the three trimesters between the repeated measures ANOVA and a standard one-way ANOVA. We thus elected to perform standard regression analysis using trimesters. (Similar results were obtained by using gestational age as a continuous variable in place of trimester grouping).

\section{Phenylalanine levels}

Regression analysis of Phe levels by trimester (Fig. 1) demonstrated a statistically significant difference (F 3, $387=1693 ; p<0.0001)$. Phe levels declined from the first trimester $(36.2+/-10.6 \mu \mathrm{mol} / \mathrm{L})$ to the second trimester $(33.4+/-9.3 \mu \mathrm{mol} / \mathrm{L})$ and then remained relatively the same in the third trimester $(32.3+/-8.7 \mu \mathrm{mol} / \mathrm{L})$. Post hoc analysis showed a significant difference between the first trimester and both the second $(p=0.046)$ and third trimesters $(p=0.007)$ but not between the second and third $(p=0.29)$.

Amino acids in the blood spot filter paper card may degrade over time, thus we studied whether this had an effect on Phe levels. Regression analysis of Phe levels against time from collection to analysis (Fig. 2) showed a slight decline of the Phe with increased time since collection $(0.004 \mu \mathrm{mol} / \mathrm{L}$ per day; $p=0.003)$ manifesting as a $4-\mu \mathrm{mol} / \mathrm{L}$ decrease for samples analyzed at 1000 days. Adjusted Phe levels were then created using the expression: adjusted Phe $(\mathrm{mmol} / \mathrm{L})=\mathrm{Phe}$ $(\mathrm{mmol} / \mathrm{L})+[($ days from collection -63$) \times 0.004]$.

Nomograms were constructed using the raw Phe level and then the adjusted Phe level (Fig. 3). A decline is evident on the nomograms, which also show an increase at the very end of the third trimester (last 30 days). Reference ranges for adjusted Phe by trimester are noted in Table 2 .

\section{Tyrosine levels}

Similar to Phe levels, regression analysis of Tyr levels by trimester (Fig. 1) demonstrated a statistically significant difference (F 3, $387=1220 ; p<0.0001)$. Tyr levels declined 

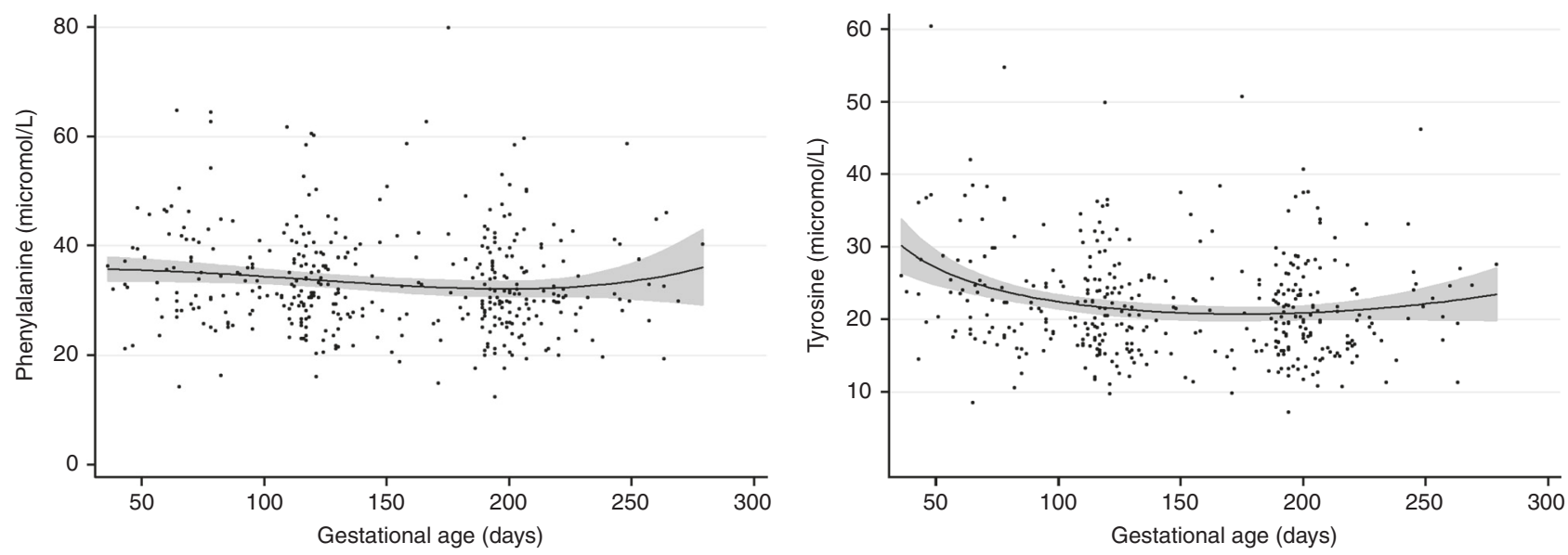

Fig. 1 Phenylalanine (left) and tyrosine (right) levels from dried blood spot cards using tandem mass spectrometry, plotted by gestational age. Line and gray shading indicate fitted quadratic line and $95 \%$ confidence intervals.
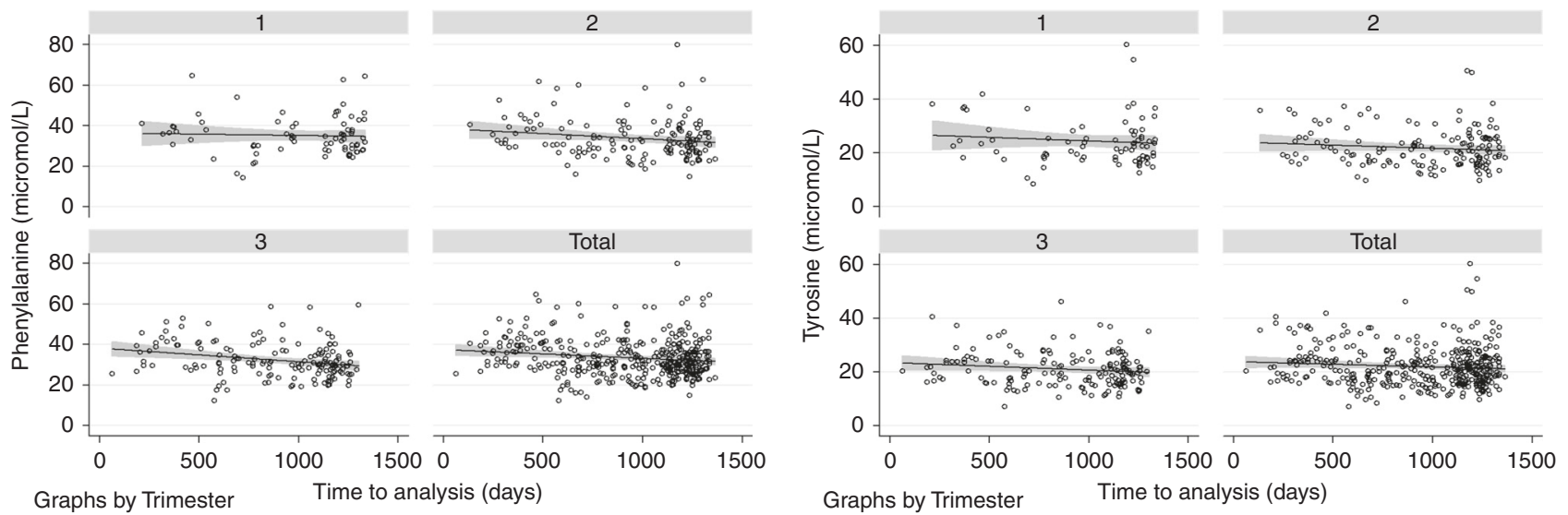

Fig. 2 Phenylalanine (left) and tyrosine (right) levels from dried blood spot cards using tandem mass spectrometry, plotted by time from collection to time analyzed, in days. Plots for first (1), second (2), and third (3) trimester are shown, along with a composite of all values from all trimesters (total). Line and gray shading indicate fitted linear regression line and $95 \%$ confidence intervals.

from the first trimester $(25.7+/-9.7 \mu \mathrm{mol} / \mathrm{L})$ to the second trimester $(21.7+/-6.7 \mu \mathrm{mol} / \mathrm{L})$ and then remained relatively the same in the third trimester $(21.0+/-6.6 \mu \mathrm{mol} / \mathrm{L})$. Post hoc analysis showed a significant difference between the first trimester and both the second $(p=0.0002)$ and third trimesters $(p<0.0001)$ but not between the second and third $(p=0.48)$.

We also assessed changes in Tyr levels based on time from collection to analysis (Fig. 2). Regression analysis showed a slight but not statistically significant decline over time $(0.002 \mu \mathrm{mol} / \mathrm{L}$ per day; $p=0.08)$, with a sample analyzed 1000 days after collection showing a $2 \mu \mathrm{mol} / \mathrm{L}$ difference. Adjusted Tyr levels were then created using the expression: adjusted $\operatorname{Tyr}(\mathrm{mmol} / \mathrm{L})=\operatorname{Tyr}(\mathrm{mmol} / \mathrm{L})+[($ days from collection -63$) \times 0.002]$.

Nomograms were constructed for both the raw $\mathrm{Tyr}$ and adjusted Tyr levels (Fig. 3). The Tyr nomograms show a quick decline from first to second trimester, and similar to Phe, an increase in the last 30 days of the third trimester. Reference ranges for adjusted Tyr by trimester are noted in Table 2.

\section{DISCUSSION}

Previous studies of amino acid levels in pregnancy have been limited to those employing AAA and serum samples, with most having very small sample sizes. ${ }^{5-11}$ This is the first study of phenylalanine and tyrosine levels in pregnant women throughout gestation using MS/MS on samples from blood spot cards. Sample sizes were large enough for us to generate the first nomograms for both Phe and Tyr levels as measured by MS/MS, providing a resource for clinicians who manage MPKU to assist in monitoring these levels.

Phe and Tyr values will differ by method and sample type. It is established that values of serum Phe and Tyr are higher than measures of Phe and Tyr from concomitantly collected blood spot samples. ${ }^{12,13}$ Values vary depending partially on sample hematocrit. In addition, many serum values of a variety of biomarkers are known to be lower in pregnancy, 

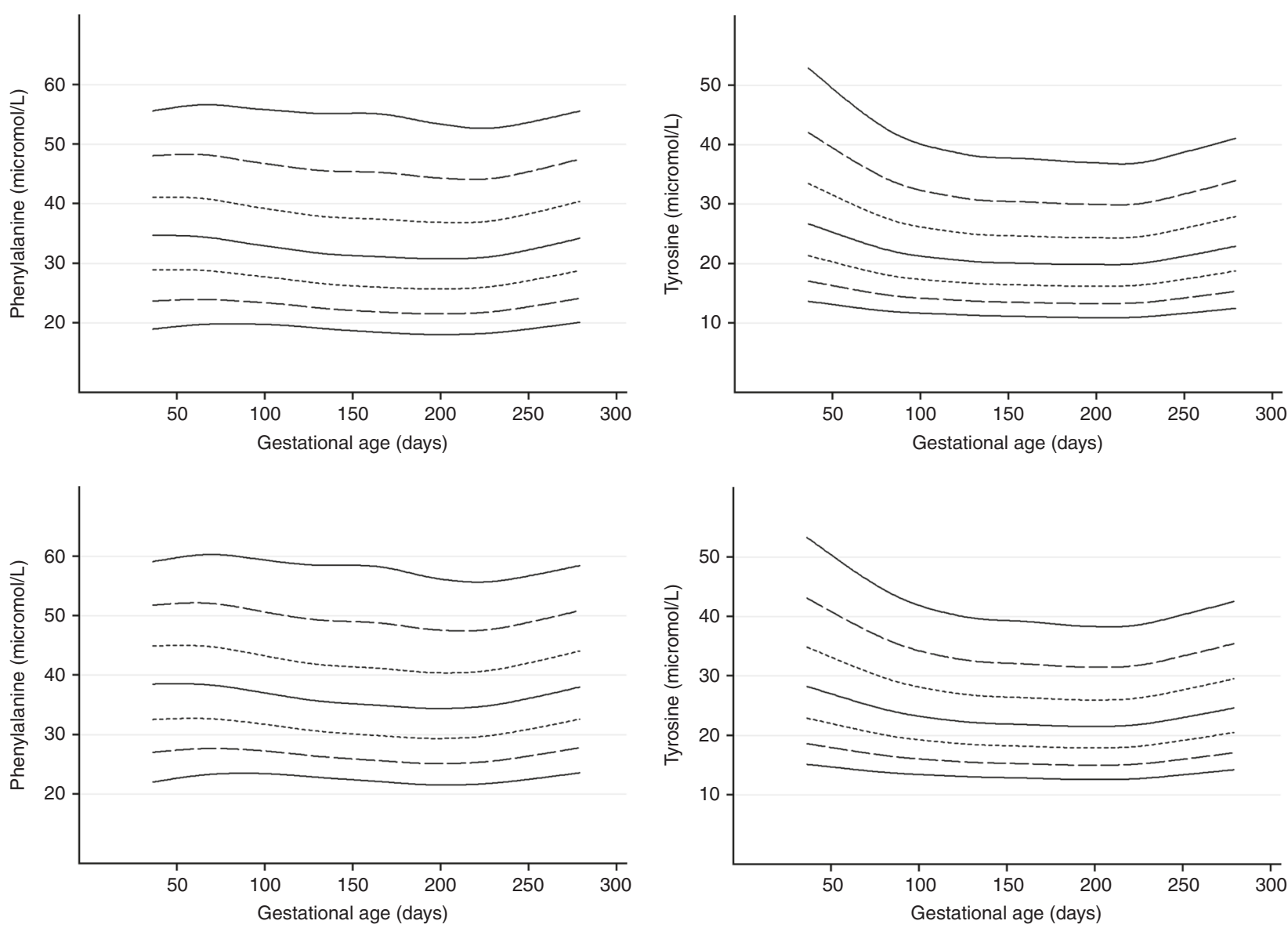

Fig. 3 Nomograms of phenylalanine (left) tyrosine (right) levels from dried blood spot cards using tandem mass spectrometry, plotted by gestational age, with raw data (top) and adjusted data for time from sample collection (bottom). Lines from top to bottom are 97th (solid), 95th (long dash), 75th (short dash), 50th (thin solid), 25th (short dash), 5th (long dash), and 3rd (solid) centiles.

Table 2 Reference ranges for phenylalanine and tyrosine ( $\mu \mathrm{mol} / \mathrm{L}$ ) during pregnancy by trimester using data adjusted for time from collection

First trimester Second trimester Third trimester

\begin{tabular}{llll}
\hline Phenylalanine & $17.9-68.4$ & $22.5-62.9$ & $22.6-57.0$ \\
Tyrosine & $10.8-59.9$ & $13.6-38.0$ & $13.1-39.2$ \\
\hline
\end{tabular}

particularly for late second and the entire third trimesters. Many factors are implicated in these changes, including increased blood volume, decreased red cell mass, fetoplacental uptake of nutrients, among others. ${ }^{15,16}$

Five previous studies have been published reporting AAA serum Phe and Tyr levels during normal pregnancy with numerical data in four of the papers. ${ }^{5-9}$ Serum Phe and Tyr levels in older studies are higher than our MS/MS values for the second and third trimester. Compared with the largest sample set, Phe levels in our study were 23\% lower and tyrosine levels were $47 \%$ lower. $^{7}$ While it is difficult to assess directly, this does show that MS/MS levels derived from blood spot cards are much lower, in line with previous observations comparing AAA with MS/MS showing differences of 15-25\% $\left(\right.$ refs. $\left.^{12,13}\right)$. In addition, serum and blood spot values also differ even when measured by the same MS/MS method. Serum Phe levels as measured by MS/MS in a study by Lindsay et al. were $14-19 \%$ higher and Tyr $30-34 \%$ higher than our blood spot values. ${ }^{10}$ This reinforces that reference ranges determined by AAA or MS/MS from serum samples should not be used for values obtained by MS/MS using blood spot cards.

Levels of both Phe and Tyr in DBS samples analyzed by MS/MS demonstrated a slight rise in the last 30 days of the third trimester. This has not been reported in previous studies of normal pregnant women. However, several studies (Table 1) have noted an increase in numerous amino acids, including Phe and Tyr, in pregnant women with preeclampsia or who are carrying a fetus with intrauterine growth restriction (IUGR). ${ }^{5,6}$ We excluded women with known chronic conditions, hypertension, pre-eclampsia, and IUGR. While it is possible some pregnancies may have had one of these conditions, we do not think this likely. Supporting this is the distribution of Phe and Tyr values for this gestational time, which do not show a skewing toward elevated levels, but a rise of values for all samples. The number of samples from this time is small, so it may be due to 
chance alone that the mean and distribution are higher. Studies of amino acid transport using maternal serum arterial and venous samples and umbilical artery and vein samples obtained at the time of delivery at term note a net Phe flux across the placenta of essentially zero, and a flux of Tyr into the maternal circulation. ${ }^{17}$ This suggests that at the end of pregnancy one might expect a return of Phe and Tyr values toward the nonpregnant levels. Whether this rise in Phe and Tyr at the end of pregnancy represents a true phenomenon will require additional investigation.

We attempted to compare values between AAA and MS/ MS, using serum samples collected at the same time as blood spots and stored in cryovials at $-80^{\circ} \mathrm{C}$ on a small subset of the pregnant women. Values for not only Phe and Tyr, but all amino acids measured by AAA, were well above our upper limits of normal for healthy adults used as reference range in our clinical lab (data not shown). We suspect volume loss and concentrating of the stored samples accounts for the higher values. Given this, we felt the data was not usable for our purposes and did not analyze and compare values between the two methods.

This study provides Phe and Tyr blood spot MS/MS reference range data by trimester (Table 2), with the full data set of centiles across gestation for both the raw data and the adjusted data in Supplemental Table 1 available for download and use by clinicians and biochemical labs. We recommend the adjusted data and centiles for lab reference ranges and nomograms for clinical practice. While the reference ranges are derived from healthy pregnant women using a reasonable sample size (1st trimester $n=78 ; 2$ nd trimester $n=152$; 3 rd trimester $n=160)$, verification by the adopting clinical lab will be important. This will be a valuable resource for care of MPKU by providing appropriate normal reference ranges for Phe and Tyr for current clinical practice of monitoring levels by blood spot cards, which will be particularly useful for assessing Phe deficiency due to overly restricted diet and possible Tyr deficiency due to undersupplementation. This will also be useful for studies on the effects of elevated or low Tyr levels in pregnancy, to better understand if deficiency is detrimental, and equally, if oversupplementation may be harmful.

\section{SUPPLEMENTRY INFORMATION}

The online version of this article (https://doi.org/10.1038/s41436018-0407-8) contains supplementary material, which is available to authorized users.

\section{ACKNOWLEDGEMENTS}

Services for this project were supported by The Ohio State University Clinical and Translational Science Award (CSTA) National Institutes of Health (NIH) grant UL1TR001070. Financial support was also received from Nationwide Children's Hospital. We thank and acknowledge the Perinatal Research Repository of the Ohio Perinatal Research Network for sharing data and samples for this project.

\section{DISCLOSURE}

The authors declare no conflicts of interest.

Publisher's note: Springer Nature remains neutral with regard to jurisdictional claims in published maps and institutional affiliations.

\section{REFERENCES}

1. Blau N, van Spronsen FJ, Levy HL. Phenylketonuria. Lancet. 2010; 376:1417-1427.

2. Levy HL, Ghavami M. Maternal phenylketonuria: a metabolic teratogen. Teratology. 1996:53:176-184.

3. Rohr FJ, Lobbregt $D$, Levy HL. Tyrosine supplementation in the treatment of maternal phenylketonuria. Am J Clin Nutr. 1998;67:473-476.

4. van Spronsen FJ, van Rijn M, Bekhof J, Koch R, Smit PG. Phenylketonuria: tyrosine supplementation in phenylalanine-restricted diets. Am J Clin Nutr. 2001;73:153-157.

5. Cetin I, Ronzoni S, Marconi AM, et al. Maternal concentrations and fetalmaternal concentration differences of plasma amino acids in normal and intrauterine growth-restricted pregnancies. Am J Obstet Gynecol. 1996; 174:1575-1583.

6. Di Giulio AM, Carelli S, Castoldi RE, Gorio A, Taricco E, Cetin I. Plasma amino acid concentrations throughout normal pregnancy and early stages of intrauterine growth restricted pregnancy. J Matern Fetal Neonatal Med. 2004;15:356-362.

7. Lopez-Quesada E, Vilaseca MA, Artuch R, Gomez E, Lailla JM. Homocysteine and other plasma amino acids in preeclampsia and in pregnancies without complications. Clin Biochem. 2003;36:185-192.

8. Ortega P, Castejon HV, Argotte MG, Gomez G, Bohorquez L, Urrieta JR. [Plasma amino acids profile of healthy pregnant adolescents in Maracaibo, Venezuela]. Arch Latinoam Nutr. 2003;53:157-164.

9. Schoengold $\mathrm{DM}$, deFiore $\mathrm{RH}$, Parlett RC. Free amino acids in plasma throughout pregnancy. Am J Obstet Gynecol. 1978;131:490-499.

10. Lindsay $\mathrm{KL}$, Hellmuth $\mathrm{C}$, Uhl $\mathrm{O}$, et al. Longitudinal metabolomic profiling of amino acids and lipids across healthy pregnancy. PLoS One. 2015;10: e0145794.

11. Orczyk-Pawilowicz M, Jawien E, Deja S, Hirnle L, Zabek A, Mlynarz P. Metabolomics of human amniotic fluid and maternal plasma during normal pregnancy. PLoS One. 2016;11:e0152740.

12. Gregory $\mathrm{CO}, \mathrm{Yu} \mathrm{C}$, Singh RH. Blood phenylalanine monitoring for dietary compliance among patients with phenylketonuria: comparison of methods. Genet Med. 2007;9:761-765.

13. Groselj U, Murko S, Zerjav Tansek M, et al. Comparison of tandem mass spectrometry and amino acid analyzer for phenylalanine and tyrosine monitoring-implications for clinical management of patients with hyperphenylalaninemia. Clin Biochem. 2015;48:14-18.

14. Jennen-Steinmetz C. Sample size determination for studies designed to estimate covariate-dependent reference quantile curves. Stat Med. 2014; 33:1336-1348.

15. Manta-Vogli PD, Schulpis KH, Dotsikas Y, Loukas YL The significant role of amino acids during pregnancy: nutritional support. J Matern Fetal Neonatal Med. 2018:1-181. https://doi.org/10.1080/14767058.2018.1489795. Accessed 3 January 2019.

16. Regnault TR, de Vrijer B, Battaglia FC. Transport and metabolism of amino acids in placenta. Endocrine. 2002;19:23-41.

17. Holm MB, Bastani NE, Holme AM, et al. Uptake and release of amino acids in the fetal-placental unit in human pregnancies. PLoS One. 2017; 12:e0185760 